\title{
Real Time Monitoring of CO2 Emissions in Vehicles Using Cognitive IOT
}

\author{
Prachi Shahane ${ }^{1}$, Preeti Godabole ${ }^{2}$ \\ ${ }^{1,2}$ SIES GST, Nerul (E), Navi Mumbai, Maharashtra, India
}

\begin{abstract}
With rise in the amount of heat trapping gases the earth is getting warmer day by day, leading to global warming. CO2 is the major contributor of the greenhouse gases. The main aim of this research is to reduce the green house effect by real time monitoring and controlling of $\mathrm{CO} 2$ emission caused due to vehicles and industries using cognitive IOT [2]. The Internet of Things (IOT) extends internet connectivity to a diverse range of devices and everyday things that utilize embedded technology to communicate and interact with the external environment, all via the Internet. In this proposal we have tried to make the CO2 detector intelligent by saving the CO2 levels in different regions. This model adheres to cognitive IOT and provides information for utilization of vehicular features based on the $\mathrm{CO} 2$ levels. The model is cost effective and also can be easily produced and integrated with vehicles and also in industries.
\end{abstract}

Keywords: Cognitive IOT, CO2 detector, Internet centric

\section{Introduction}

The life and health of all living beings are correlated to the prevailing climatic conditions. A warming climate will bring changes that can affect our water supplies, agriculture, power and transportation systems, the natural environment, and even our own health and safety. Certain changes to the climate are unavoidable. Carbon dioxide can stay in the atmosphere for nearly a century, so Earth will continue to warm in the coming decades. The warmer it gets, the greater the risk for more severe changes to the climate and Earth's system. Greenhouse Gas Emissions council has done a survey in 2013 says CO2 has a major contribution of $82 \%$ of the total green gases emitted. And the statistics also says $27 \%$ of the green gases is due to transportation and 31\% is from industries.[1] India is at fourth position and contributing $8.95 \%$ of the total $\mathrm{CO} 2$ emissions in the entire world. The survey of $\mathrm{CO} 2$ emissions from vehicles in India reveals that major vehicle contributor of $\mathrm{CO} 2$ are cars, taxis and MUV's. The proposed design is hence based on the cars and taxis.

Although it's difficult to predict the exact impact of climate change. It is clear that the climate we are accustomed to is no longer a reliable guide for what to expect in the future. These risks from climate change to the human race can be reduced to a large extent [2][3]. By making choices that reduce greenhouse gas pollution, and preparing for the changes that are already underway, we can reduce risks by continuous monitoring of $\mathrm{CO} 2$ emissions.

The new concept of the Cognitive Internet of Things (CIOT) brings an opportunity for the creation of innovative applications that integrate the all too familiar traditional digital technologies. The CIOT is about interfacing these autonomous devices to communicate without human intervention and generate integrated data. Intelligence is then required to process this integrated data and make it available to the humans for decision-making. [4] This concept of CIOT has been applied to monitor greenhouse gases released by the vehicles. CO2 sensors are available but no system is available for real time monitoring and thus controlling the $\mathrm{CO} 2$ emission levels with the help of the control center. The amount of CO2 emissions have to be reduced by having a check on the transport system or industries with the help of governing bodies. The proposed model helps in real time monitoring of $\mathrm{CO} 2$ emissions. The decision making can happen at the central server at the environment board or the Pollution control board. CO2 can be controlled and thus helps in reducing global warming.

\section{Literature Survey}

In the Internet of Things (IOT) paradigm, many day to day objects that are around us will be on a network in some form or another and first was used in context of supply chain management [5]. The definition was reframed and included a wide range of applications like healthcare, utilities, transport, etc [6]. The main goal of making computer sense information without the aid of human intervention is the gist of IOT. IOT is based on harvesting information from the environment (sensing) and interact with the physical world (actuation/command/control). It uses existing Internet standards to provide services for information transfer, analytics, applications, and communications. Various open wireless technologies have boosted the development of IOT such as Bluetooth, radio frequency identification (RFID), Wi-Fi, and telephonic data services as well as embedded sensor and actuator nodes [7]. The research on Cognitive Internet of Things (CIOT) is very limited. A cognitive management framework is presented to empower the IOT to better support sustainable smart city development. Cognition mainly refers to the autonomic selection of the most relevant information for the given application. CIOT is viewed as the current IOT integrated with cognitive and cooperative mechanisms to promote performance and achieve intelligence [3]. The survey reveals that total CO2 emissions from an average car showed that $76 \%$ were from fuel usage where as $9 \%$ was from manufacturing of the vehicle and a further $15 \%$ was from emissions and losses in the fuel supply system[2]. 


\section{International Journal of Science and Research (IJSR) \\ ISSN (Online): 2319-7064}

Index Copernicus Value (2013): 6.14 | Impact Factor (2014): 5.611

\section{Methodology}

The proposed design falls in the CIOT framework. The framework of CIOT, serves as a transparent bridge between physical world like objects, and social world together with itself to form an intelligent system. [4] The cognitive process of the our system where $\mathrm{CO} 2$ in the vehicle exhaust is monitored consists of four major layers:

1)Sensing control layer: This layer has been directly interfaced with physical environment, in which the MG811 sensor senses the CO2 gas. The signals are generated if CO2 levels are beyond expected levels. These signals are sent to the upper layer.

2)Data-semantic-knowledge layer: In this layer the data from the sensor control layer is put in the data base and efficiently analyzed. This makes it useful semantic and is then added to the knowledge base.

3)Decision-making layer: It uses the knowledge abstracted from the lower semantic layer to make the decisions. The decisions in our system are sending of alerts to the owner and central control board. These decisions are made based on the abstracted data.

4) Service evaluation layer: This layer shares important interfaces with social networks and automobile industries in which feedback and the evaluation result forms the cognition process.

The vision of IOT can be seen from two perspectives Internet centric and Thing centric. The Internet centric architecture will involve internet services being the main focus while data is contributed by the objects. In the object centric architecture, the smart objects take the center stage. In our work, we develop an Internet centric approach [8]. The entire model is divided in to two sub models as shown in figure 1.

1. Central Board module on the cloud server and payment gateway.

2. Owner side module with PI and CO2 sensor.

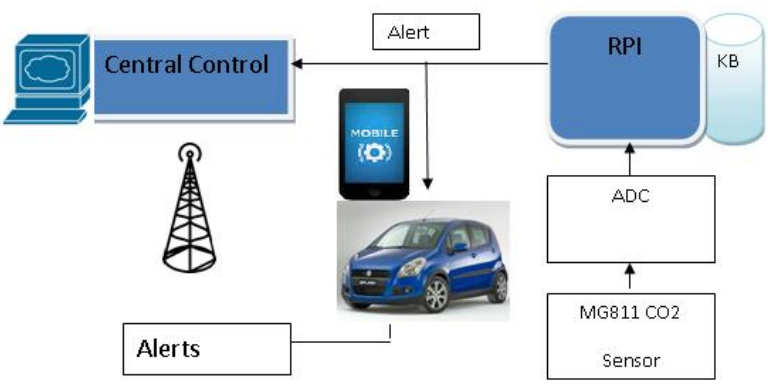

Figure 1: Proposed Model of the detector in vehicles

\subsection{Central Board Module}

Billions of smart objects will be immersed in the environment, sensing, interacting, and cooperating with each other to enable efficient services that will bring tangible benefits to the environment, the economy and the society as a whole. The central board receives alerts and respective credentials from millions of vehicles. The amount of data collected at the central control is very huge. The most cost effective solution to this is cloud. The data generated at the central board has to be put on the cloud. The data at the central server is abstracted using Data Analytics Algorithm. The abstracted data can reveal the number of alerts of a particular vehicle over a period of time say one month. If the alerts of a particular vehicle exceeded the threshold over a period of time, heavy fine can be levied on the owner via online payment gateway. The abstracted data also provides service to social networks and also to automobile industries for evaluation of the vehicles.

\subsection{Owner Module}

The car owner has to install a designed $\mathrm{CO} 2$ detector at the exhaust to sense the level of $\mathrm{CO} 2$ emitted in terms of PPM. The CO2 sensor is communicating with the PI in the vehicle. If the $\mathrm{CO} 2$ level exceeds the normal PPM level (600 PPM) [3] the PI sends a notification to the registered mobile of the owner. This notification is like a warning message given to the owner so that owner is allowed to rectify the vehicle.

If the owner does not take any action to reduce the emissions, a alert is sent to the central board via GSM module on the PI. The alert message consists of the user information like the GSM number of the PI and credentials of the owner including bank account number. The manufacturer of the car is also sent to the central board with the credentials which can be further used for automobile analysis. This data is acts as useful semantic to take decisions about the manufacturer at the central board. The decision taken by central board becomes a major step in reducing greenhouse effect. If the alerts at the central board exceed threshold (T1) then the board sends an alert to the owner using the registered credentials. The average of the alerts taken over a period of time exceeds threshold (T2) then automatically it connects to the payment gateway and penalty will be levied on the vehicle owner.

The flow diagram in figure 2 shows the functioning of the $\mathrm{CO} 2$ detector. 


\section{International Journal of Science and Research (IJSR) \\ ISSN (Online): 2319-7064}

Index Copernicus Value (2013): 6.14 | Impact Factor (2014): 5.611

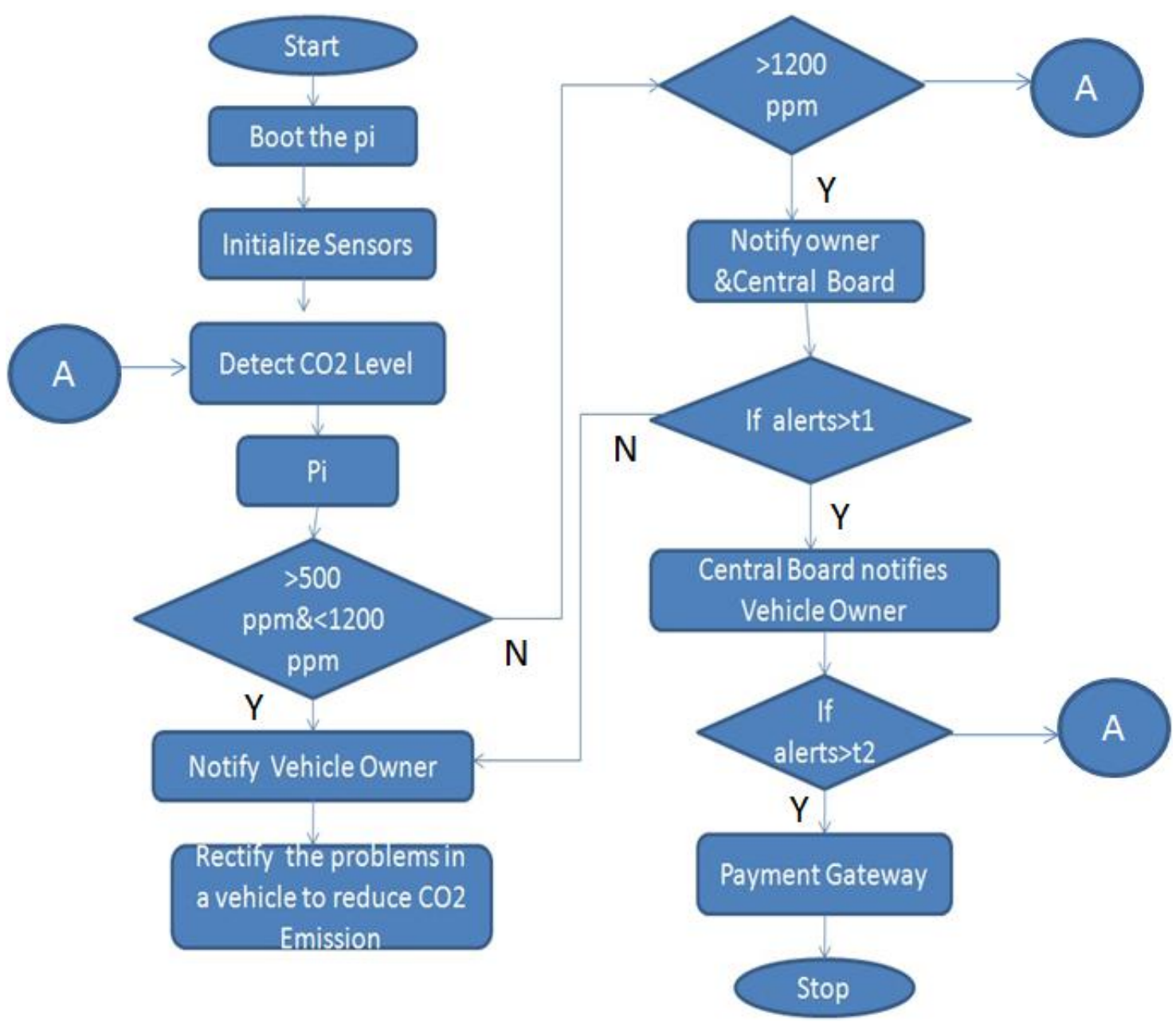

Figure 2: Flow diagram of the model

\subsection{CO2 Sensors}

Various CO2 sensors may be classified in two major categories depending on measuring principle of sensors. First, the chemical $\mathrm{CO} 2$ gas sensors have the principal advantage of a very low energy consumption and small size. Second, NDIR-based CO2 sensor is commonly used in monitoring indoor air quality due to relatively high accuracy compared with that of a chemical $\mathrm{CO} 2$ gas sensor.[14] MG811 works on solid electrolyte cell principle. MG811 is highly sensitive to $\mathrm{CO} 2$ and compatible with Raspberry Pi. The MG811 CO2 (Carbon Dioxide) Gas Sensor is used in gas detection equipment for detecting Carbon Dioxide gas in automotive or industrial settings. MG811 has a wide measuring range of $300 \mathrm{ppm}$ to $10000 \mathrm{ppm}$. And this range is required to measure the acceptable levels of CO2 in the air. MG811 has analog output 30 50mV, (higher consistency, higher voltage) of carbon dioxide with high sensitivity and good selectivity. It has Long life span and reliable long-term stability. MG811 has a sensor removable design which is convenient for testing. The operating range of the sensor is -4 to $50^{\circ}[13]$.

MG811 gas sensor responds to $\mathrm{CO} 2$ gas by generating a small voltage in proportion to the amount of $\mathrm{CO} 2$ gas present in the air exposed to the internal element. The sensor is a high impedance device and requires a buffer/amplifier to measure the output. The output of opamp through a resistor providing a TTL-compatible signal to a microcontroller. The voltage from the sensor actually drops as CO2 increases. The sensor is easily calibrated by breathing. Humans also exhale CO2. Exhaling once at the sensor leads to change in voltage levels.

The Sensor is very sensitive that it can sense the CO2 emitted from other vehicles while stuck in Traffic which can disturb the integrity of the system. This can be overcome by reading the samples over a period of time and then based on the average value the system will take an action.

\section{Future Scope}

The proposed model detects only emission of carbon dioxide. But, there are many harmful gases which pollute the environment like carbon monoxide, methane, nitrous oxide etc. The prototype can be extended to detect these gases which cause harm to our precious environment. The sensor currently used is MG811 for detection of CO2 emissions. It can withstand a temperature up to 70 degrees. A high grade sensor can be used instead of MG811 to withstand higher temperatures, so that the entire system can be installed in the exhaust of the vehicle. The prototype can not only be used in vehicles but also in 


\section{International Journal of Science and Research (IJSR) \\ ISSN (Online): 2319-7064 \\ Index Copernicus Value (2013): 6.14 | Impact Factor (2014): 5.611}

many industries to measure the harmful gases to reduce air pollution caused due to these gases.

\section{Conclusion}

Pollution Check has to be done every 6 months and hardly people get it done. Our model has to be installed one time and goes on for 10 years. Also the model adheres to real time monitoring of $\mathrm{CO} 2$ which can reduce the greenhouse gas in the environment as compared to the existing system of pollution check. This Product can cut down and control the emission considerably. It will gradually reduce Global Warming, if implemented on global scale.

\section{References}

[1] "Greenhouse gas emission reduction scenarios for bc",Colin R. Campbell and Cliff Stainsby, Copublished by the Canadian Centre for Policy Alternatives - BC Office, the Sierra Club of BC, and the BC Government and Service Employees' Union.

[2] "What will global annual emissions of greenhouse gases be in 2030, and will they be consistent with avoiding global warming of more than $2^{\circ} \mathrm{C}$ ?", Rodney Boyd, Nicholas Stern and Bob Ward, Policy paper May 2015, ESRC Centre for Climate Change Economics and Policy Grantham Research Institute on Climate Change and the Environment

[3] "Research Directions for the Internet of Things", John A. Stankovic, Life Fellow, published in March 2014, in Internet of Things IEEE journalwith ISSN: 23274662

[4] "Cognitive Internet of Things: A New Paradigm beyond Connection", Qihui Wu, Senior Member, IEEE, Guoru Ding, Student Member, IEEE, Yuhua $\mathrm{Xu}$, Student Member, IEEE, Shuo Feng, Zhiyong Du, Jinlong Wang, Senior Member, IEEE, and Keping Long, Senior Member, IEEE, arXiv:1403.2498v1 [cs.AI] 11 Mar 2014

[5] The Greenhouse Effect" Wicked Danger - or Merely a Hoax? ", Klaus Ermecke and Heinz Thieme K. Ashton, That -Internet of Things\| Thing, RFiD Journal. (2009).

[6] International Journal of Scientific \& Engineering Research, Volume 4, Issue Ş, 2013 ISSN 2229-5518 IJSER (C) 2013 http://www.ijser.org Measurement of atmospheric Carbon Dioxide using multi sensor approach Akshay Anant Bhide, S.L. Nalbalwar

[7] J. Buckley, ed., The Internet of Things: From RFID to the Next-Generation Pervasive Networked Systems, Auerbach Publications, New York, 2006

[8] "Internet of Things (IOT): A Vision, Architectural Elements, and Future Directions", Jayavardhana Gubbi,a Rajkumar Buyya,b* Slaven Marusic,a Marimuthu Palaniswamia a Department of Electrical and Electronic Engineering, The University of Melbourne, Vic - 3010, Australia b Department of Computing and Information Systems, The University of Melbourne, Vic - 3010, Australia, www.elsevier.com/locate/fgcs 29(2013)1645-1660

[9] H. Sundmaeker, P. Guillemin, P. Friess, S. Woelfflé, Vision and challenges for realizing the Internet of
Things, Cluster of European Research Projects on the Internet of Things - CERP IOT, 2010.

[10]P. Vlacheas, R. Giaffreda, V. Stavroulaki, et al, Enabling smart cities through a cognitive management framework for the internet of things," IEEE Communications Magazine, vol. 51, no. 6, pp. 102-111, June 2013

[11] Transport and climate change: a review Lee Chapman * www.elsevier.com/locate/jtrangeoJournal of Transport Geography 15 (2007) 354-367

[12] http://www.epa.gov/climatechange/ghgemissions/sour ces.html

[13] http://co2meters.com/Documentation/Datasheets/DS 30-01\%20-\%20K30.pdf , Revision 1.3 - May 2015

[14]"A study on NDIR-based CO2 sensor to apply remote air quality monitoring system", Jongwon Kwon ; Gwanghoon Ahn ; Gyusik Kim ; Jo Chun Kim Published in: ICCAS-SICE, 2009 Page(s):1683 1687, Print ISBN:978-4-907764-34-0 INSPEC Accession Number: 10982401 